Projets

de paysage

\section{Projets de paysage}

Revue scientifique sur la conception et l'aménagement de l'espace

$17 \mid 2017$

Paysage(s) et agriculture(s)

\title{
Landwirtschaft auf stadtnahen Flächen
}

Erkundungen auf dem Reyerhof in Stuttgart-Möhringen

Voyage en terres urbaines cultivées - Au Reyerhof, à Stuttgart-Möhringen

\section{Dominique Henry}

Traducteur : Daniela Weber

\section{OpenEdition \\ Journals}

Édition électronique

URL : http://journals.openedition.org/paysage/4634

DOI : 10.4000/paysage.4634

ISSN : 1969-6124

\section{Éditeur :}

École nationale supérieure du paysage de Versailles-Marseille, Institut national des sciences appliquées Centre Val de Loire - École de la nature et du paysage, École nationale supérieure d'architecture et de paysage de Bordeaux, École nationale supérieure d'architecture et de paysage de Lille, Agrocampus Angers

\section{Référence électronique}

Dominique Henry, «Landwirtschaft auf stadtnahen Flächen », Projets de paysage [En ligne], 17 | 2017, mis en ligne le, consulté le 03 avril 2020. URL : http://journals.openedition.org/paysage/4634 ; DOI :

https://doi.org/10.4000/paysage.4634

Ce document a été généré automatiquement le 3 avril 2020.

Projets de paysage 


\title{
Landwirtschaft auf stadtnahen Flächen
}

\author{
Erkundungen auf dem Reyerhof in Stuttgart-Möhringen \\ Voyage en terres urbaines cultivées - Au Reyerhof, à Stuttgart-Möhringen
}

\section{Dominique Henry}

Traduction : Daniela Weber

\section{NOTE DE L'AUTEUR}

Unter freundlicher Mitwirkung von Lukas Dreyer, Landwirtschafts- und Gärtnermeister, Komplementär der Reyerhof KG und Alina Reinartz, ArchitektinStadtplanerin und Landwirtin, Gründungsmitglied der SoLaWiS (Solidarische Landwirtschaft Stuttgart).

\section{„Landwirtschaft in der Stadt für die Stadt"}

1 Stadt und Landwirtschaft, beide benötigen Raum. Das ist eine Tatsache. Für beide gilt : um zu wachsen, müssen sie sich ausdehnen. Nur: Was für die Stadt Bauland darstellt, ist für die Landwirtschaft der zu bewirtschaftende Boden. Auch das ist somit eine Tatsache : für die beständige Ausbreitung der Städte werden der Landwirtschaft (und dem Wald) Flächen entzogen. So schwellen die Städte an, dehnen sich aus und wachsen, aber manchmal umkreisen sie auch die landwirtschaftlichen Flächen, umfangen sie und schließen sie ein. Denn die Urbanisierung ist, abgesehen von der dichten Bebauung der alten Stadtkerne, kein kontinuierliches und homogenes Gewebe aus Gebäuden und Infrastrukturen. Eher handelt es sich zumeist um ein lockermaschiges räumliches Phänomen, das einen ausgesprochen hohen Bedarf an Boden hat und Landschaften hervorbringt, die wir oft nur schwer beschreiben können - und manchmal wenig schätzen. Dies kommt daher, dass wir unsere gewohnten Analyseraster zugrunde legen, welche noch immer weitgehend auf der Vorstellung einer klaren Aufteilung zwischen 
der kompakten Stadt einerseits und den landwirtschaftlich geprägten ländlichen Gegenden andererseits beruhen. Die Stadterweiterungen haben stets ein komplexes Erscheinungsbild. Ihre Formen werden bisweilen als tentakelartig beschrieben, als falsch zusammengesetzte Puzzleteile, als eine Wolke Konfetti oder als eine lückenhafte Fläche, in der „landwirtschaftliche Enklaven“ fortbestehen („enclaves agricoles“, Janin, 2009).

2 Diese Erweiterung des Bereichs Städtebau („extension du domaine de l'urbanisme“, Masboungi, 2014) erzeugt eine große Vielfalt an Kontakten, Annäherungen, neuen Nachbarschaftsbeziehungen - ausgehandelt oder nicht - zwischen der Stadt und ihrer landwirtschaftlich geprägten Umgebung. Zumeist geben die Landwirte dem Druck nach und sehen sich gezwungen, das Feld zu räumen und zurückzuweichen oder andernfalls sih anzupassen. Aber zugleich entstehen auch Initiativen - ob öffentlicher oder privater Natur - , die versuchen, die Aufteilung des Raums auszuhandeln, vorauszuplanen und zu festigen. Dies ist beispielsweise der Fall in Großstädten, die Rücksicht auf die landwirtschaftlichen Flächen nehmen durch Konzepte wie den Agrarpark in der Toskana (Bernetti et al., 2008) und die "Champs urbains" (,urbane Felder") im französischen Rennes (Boutleux, 2013) oder durch Vereine wie „Triangle vert“ („Grünes Dreieck“) $)^{1}$, hervorgegangen aus dem Zusammenschluss von gemüsebaulich geprägten Städten der Landschaft Hurepoix im Ballungsraum Paris (Laverne, 2011). Daneben - jedoch weniger bekannt als solche öffentlichen Projekte gibt es auch Landwirte, die versuchen, sich anzupassen, der Entwicklung entgegenzutreten und ihre wichtige eigene Rolle, ja die Notwendigkeit ihrer Existenz zu bekräftigen. Jene Landwirte ziehen Nutzen aus ihrer urbanen Lage und entwickeln verschiedene Annäherungen an die Stadt. Da jeder landwirtschaftliche Betrieb in Großstadtnähe seine eigene Geschichte hat, wäre es interessant, die jeweiligen Ansätze und Strategien herauszuarbeiten, die zugehörigen Agrarlandschaften zu untersuchen sowie die Landwirte und betroffenen Verbraucher zu befragen. Dabei ginge es darum, die Landschaften vor Ort zu erforschen und dieses Konzept der Annäherungen, die zwischen Landwirten und Städten zu entstehen scheinen, besser zu erfassen. Damit ließe sich gewiss etwas beitragen zum von Rémi Janin angestrebten Landwirtschaftsprojekt für die Stadt; er hat hierzu ein anregendes Essay formuliert (Janin, 2017). Dieser Aspekt könnte eine interessante Perspektive für zukünftige Landschaftsforschungen bilden. In manchen Arbeiten kommt dieses Themenfeld bereits zur Sprache (Toublanc und Poulot, $2017^{2}$ beispielsweise).

3 Gegenstand des vorliegenden Beitrags ist es, in bescheidenerem Maße den Blick auf die Agrarlandschaften $\mathrm{zu}$ richten, die durch einen Landwirtschaftsbetrieb im engen Kontakt mit einem Ballungsraum wie Stuttgart erzeugt wurden. Es handelt sich um den Reyerhof ${ }^{3}$, einen biologisch-dynamischen Gemischtbetrieb, auf dem ich mich regelmäßig aufhalte und in dessen Laden ich einkaufe. Der Slogan des Reyerhofs "Landwirtschaft in der Stadt für die Stadt" hat in mir das Interesse geweckt, seine landwirtschaftlich genutzten stadtnahen Flächen zu erkunden und an dieser Stelle davon zu berichten. Dieser Werbespruch, der auf dem Lieferwagen und den Werbungen steht, bringt sowohl ein Verkaufsargument zum Ausdruck als auch einen tatsächlichen Sachverhalt. Denn die vom Reyerhof bewirtschafteten Parzellen fügen sich in das verstädterte Gebiet von Stuttgart ein, sie befinden sich am Rand der Stadtviertel, genauer gesagt zwischen Möhringen und den anderen Gemeinden, die den südlichen Teil dieses Ballungsraums bilden. Als gelegentlicher Bewohner des Hofes und 
Verbraucher seiner Produkte ist in mir das Interesse erwacht, die Landschaften und Flächen kennenzulernen, die zum Hof gehören und von denen das Gemüse kommt, das ich gern esse.

4 Der hier ausgeführte Gedanke der Erkundung ${ }^{4}$ drückt den Wunsch aus, durch eine Fußwanderung die Begegnung ${ }^{5}$ mit den Agrarlandschaften und mit denjenigen, die sie bewirtschaften, zu suchen. Dabei handelt es sich um Lukas, Landwirt und Gemüsebauer, Komplementär der Reyerhof KG, mit dem ich gesprochen habe, und um Alina, die zunächst ein Architekturstudium abgeschlossen und dann (unter anderem auf dem Reyerhof) eine landwirtschaftliche Lehre absolviert hat. Als Landwirtin ist sie seit kurzem Angestellte auf dem Hof. Sie ist Gründungsmitglied der SoLaWi Stuttgart ${ }^{6}$. Aus praktischen Gründen hat Letztere mir einen Text übermittelt, in dem sie meine Fragen beantwortet hat. Die daraus zitierten Passagen sind Teil des Berichts und bringen zweierlei Sichtweisen - der Architektin und der Landwirtin - auf die Beschreibung der Landschaften zum Ausdruck. Schließlich, weil ich bei meinen Wanderungen zugleich zeichne, was eine individuelle, eher allein ausgeübte Aktivität ist, muss ich dazu sagen, dass die beiden Formen der Begegnung (mit den Landschaften einerseits und mit den Landwirten andererseits) getrennt voneinander stattgefunden haben, wobei jedoch unter ihnen eine Verbindung durch Dokumente bestand (Landkarten und Zeichnungen), die als Grundlage der Gespräche gedient haben.

Die Landschaften dieses großstädtischen Landwirtschaftsbetriebs $\mathrm{zu}$ erfassen, $\mathrm{zu}$ beschreiben und darzustellen, darin bestand für mich das Ziel dieser Erkundung und dieses Beitrags, in welchem Material zusammengetragen wird, aus dem potenziell eine breiter angelegte Forschungs- und Projektarbeit hervorgehen könnte.

\section{Ankunft auf dem Reyerhof, erste Kontakte mit den Landschaften}

\section{Ganz in der Nähe des Stadtzentrums von Stuttgart}

6 Vom Schlossplatz aus - einem der Plätze im Stadtzentrum von Stuttgart - fährt man mit der Stadtbahnlinie U $5^{7}$ nicht mehr als eine Viertelstunde bis Rohrer Weg, der vom Bauernhof aus nächstgelegenen Haltestelle. Aus dem Kessel, in dem sich das Stadtzentrum von Stuttgart befindet, erklimmt die Stadtbahn den steilen Hang und gelangt dann zu der Hochebene, auf der Möhringen liegt. Denn die Gemeinde befindet sich ganz oben im Einzugsgebiet der Körsch, dort wo die Ebene, die sich über die linke Seite des Neckartals erhebt, ausgehöhlt ist und verschiedene Wasserläufe zusammenfließen, um dann das Körschtal zu bilden. Die auf einer Höhe von etwa 400 Metern liegende, leicht hügelige Landschaft beschrieb André Birre 1969 folgendermaßen: „ein 8 Meter dicker lehmiger Boden, frisch und fruchtbar, gut geeignet für den Anbau von Kohl für Sauerkraut, für welches das Land berühmt ist“. Auch heute wird hier noch viel Kohl angebaut, und im Sommer kann man am Rande von Möhringen die weiten, bläulich gefärbten Felder überblicken.

7 Kurz vor der Haltestelle Rohrer Weg fährt die Stadtbahn langsamer. Da bemerke ich sie, durch die Fenster des U-Bahn-Wagens. Zuerst sehe ich jede Menge Fahrräder am Feldrand, Plastikkisten im Gras hinter dem Lieferwagen mit der Aufschrift , Landwirtschaft in der Stadt für die Stadt", und dann sie : fünf bis sechs Personen, die Füße im Stroh, gebeugt oder in der Hocke zwischen den Erdbeerreihen. Später werde ich 
erfahren, dass Erntetag ist. Angestellte, Betriebsleiter und Lehrlinge des Hofes sind eifrig dabei, die wertvollen roten Früchte in Schalen aus recycelter Pappe zu legen. Das Wochenende steht vor der Tür, und der Erdbeervorrat für den Hofladen muss aufgestockt werden, während der Rest der reichen Ernte zu Erdbeerpüree zur Aromatisierung von auf dem Hof hergestelltem Eis und Joghurt verarbeitet wird.

\section{„Haltestelle Rohrer Weg“}

Abbildung 1. Rohrer Weg, ein landwirtschaftlicher Raum im Blickfeld, durch den die Stadtbahn am Rand von Stuttgart-Möhringen fährt

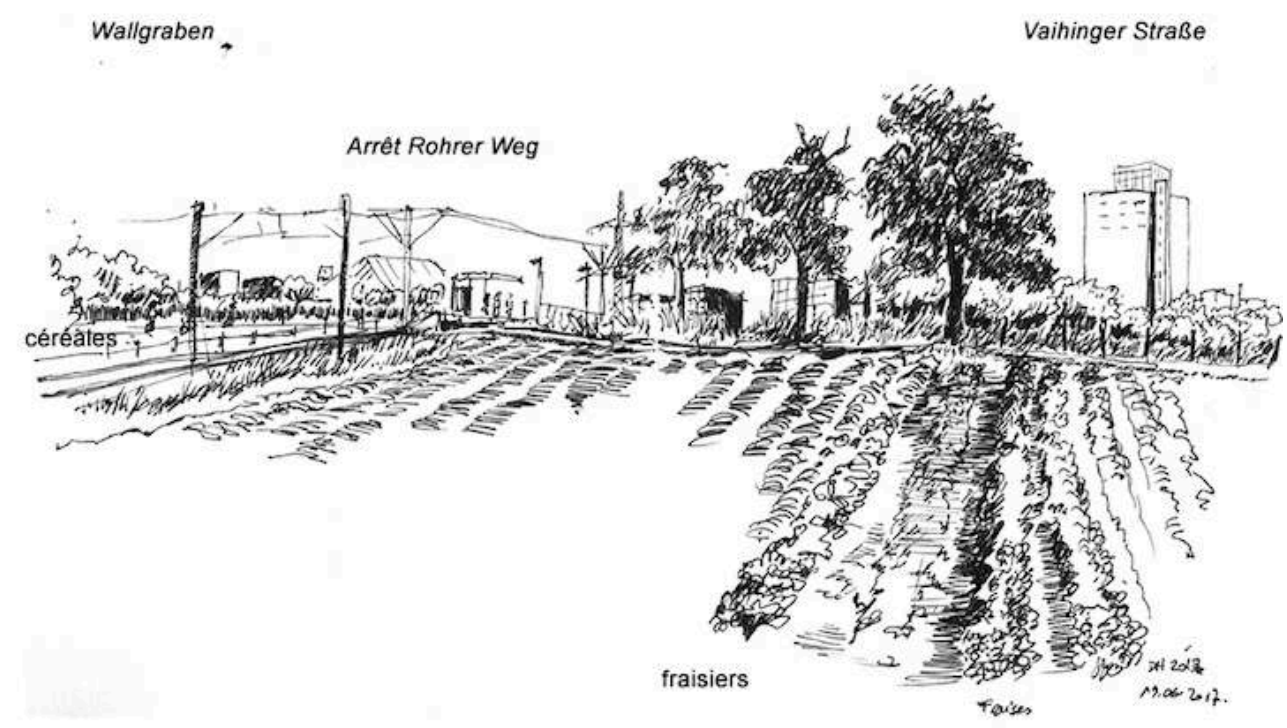

Dominique Henry, 2017

Die Besonderheit der Haltestelle, an der ich aussteige, besteht darin, dass sie buchstäblich inmitten von Feldern liegt. Es handelt sich schlicht und ergreifend um einen Bahnsteig mitten in einem landwirtschaftlich genutzten Gebiet, welches so ausgedehnt ist, dass man von hier aus weit in die Landschaft blicken und in gewisser Entfernung die verschiedenen Viertel im Westen von Möhringen sehen kann. Ein Rundumblick zeigt im Osten einen Horizont, der von den Dächern des Ortskerns von Möhringen begrenzt und von der Skyline der Wohnblocks von Fasanenhof überragt wird, während sich auf der Westseite über dem Laubwerk die Gebäude des Dienstleistungsstandortes Wallgraben erheben.

9 Kurz vor meiner Ankunft auf dem Reyerhof befinde ich mich hier im Herzen der Landschaften, die von diesem Betrieb im Süden Stuttgarts miterzeugt werden ; sie sind gekennzeichnet durch einen landwirtschaftlichen Raum, in dem sich Hackfruchtkulturen, Getreidefelder und Weiden zwischen Obstwiesen mit alten Apfelbäumen abwechseln. Diese Landschaften, die das Ergebnis eines direkten Kontakts zwischen gewöhnlich so unterschiedlichen Wirklichkeiten sind - verschiedene Anbaupflanzen, Feldarbeiter vor einer Stadtkulisse -, erstaunen und begeistern mich. Diese Art von üppiger Vegetation und räumlicher Nähe im Zusammenhang mit einer 
„bäuerlichen“ Landwirtschaft und Gemüseanbau gibt es kaum in oder am Rande von französischen Städten wie beispielsweise Bordeaux, Straßburg, Lille oder Niort, wo eher eine Landwirtschaft auf größeren Flächen vorherrscht. Diese Konstellation ist so ungewöhnlich, dass sie meine Aufmerksamkeit geweckt und mich zur Erkundung der vom Hof bewirtschafteten Ackerflächen angeregt hat. Deren räumliche Verteilung veranlasst mich, einmal rund um die Möhringer Gemarkung zu wandern.

Diese Idee eines „Rundgangs ums Dorf“ sagt mir durchaus zu. Denn sie erscheint mir wie eine Variante der „Flurbegehung“, einer Praxis, die für Landwirte und Agronomen von großer Bedeutung ist (Deffontaines, 1998) und auch für einige Landschaftsarchitekten (Ambroise et al., 2000 ; Henry, 2004), um sich ein Bild über den Zustand der Kulturen oder der Landschaft $\mathrm{zu}$ machen und $\mathrm{zu}$ entscheiden, welche Maßnahmen oder Projekte umgesetzt werden sollen.

\section{Rundgang um Möhringen}

Abbildung 2. Erkundungen der Landwirtschaft auf stadtnahen Flächen, bewirtschaftet durch den Reyerhof, rund um Möhringen

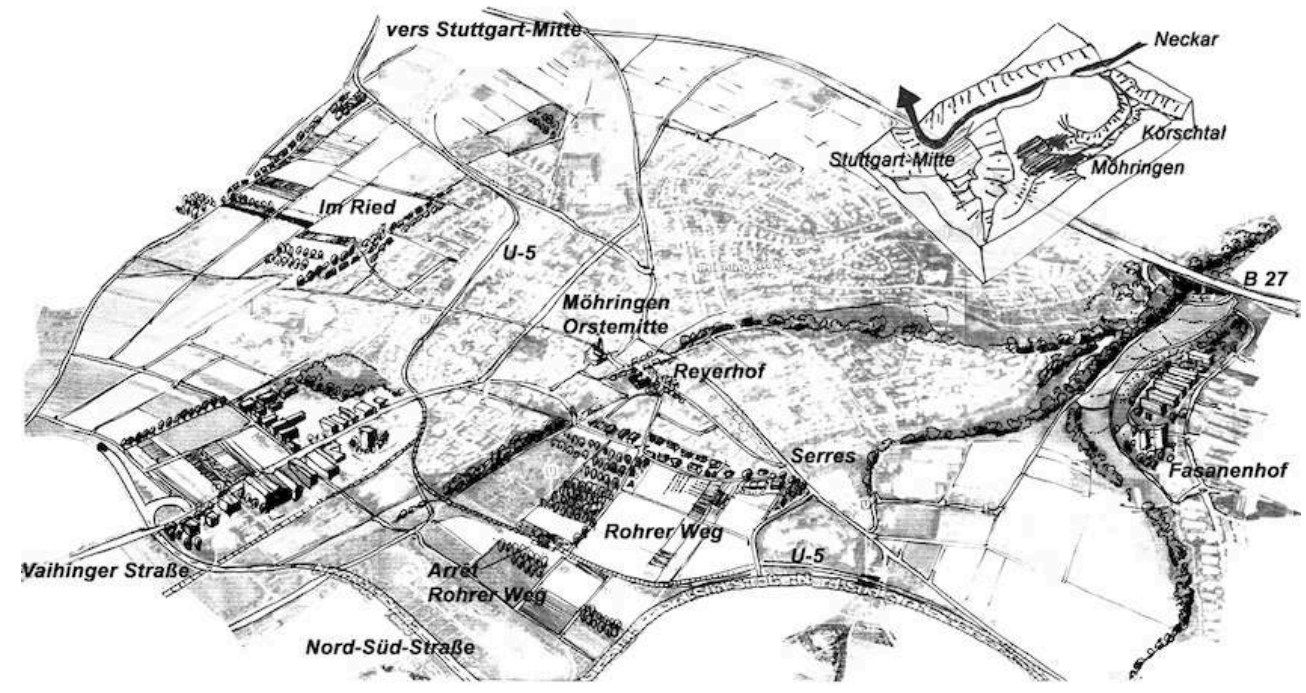

Dominique Henry, 2017

Wenn man die Landschaft aus der Luft betrachtet, erfasst man die Verschachtelung von Feldern und bebautem Raum, welche für diese Landwirtschaft in der Stadt bestimmend ist. Der landwirtschaftliche Raum erscheint hier umgrenzt, eingeschlossen inmitten einer städtischen Siedlung mit ihrem komplexen Erscheinungsbild aus verschiedenen Verkehrsinfrastrukturen, Gebäudearten und Bebauungsdichten. Im Norden, im Süden wie im Westen grenzen die landwirtschaftlichen Flächen direkt an Bauten mit sehr unterschiedlicher Höhe, Anordnung, Funktion und Atmosphäre. Da ich die Kontraste der Stadtlandschaft in ihrer engen und frontalen Beziehung zur Organisation der vielfältigen Kulturen erfassen möchte, lege ich den folgenden Verlauf meiner Erkundungstour fest: ich suche nacheinander vier Flurstücke auf - Rohrer Weg, Vaihinger Straße, Im Ried sowie Fasanenhof, um schließlich im Hof des Reyerhofs zu enden. 


\section{Rohrer Weg}

12 Neben der Durchfahrt der Stadtbahnlinie zeichnet sich dieses Flurstück dadurch aus, dass südlich und westlich davon die Nord-Süd-Straße entlangführt (Hauptverkehrsstraße mit Anbindung an die Autobahn A8) und dass es östlich und nördlich von einem Gefüge aus Wohnbauten begrenzt ist, bestehend aus Einfamilienhäusern und kleinen Mehrfamilienhäusern. Das Gebiet wird von einem Netz von Feldwegen durchzogen (zugänglich für die Öffentlichkeit), es handelt sich um einen landwirtschaftlichen Raum, der nicht nur sehr gut sichtbar ist, sondern auch sehr belebt, sowohl für Freizeitaktivitäten als auch im Rahmen der Alltagsmobilität. Die Landschaft ist extrem kleinparzelliert. Dies trägt zum Nebeneinander verschiedener Kulturen bei (Getreide, Erdbeeren, Kartoffeln, Schnittblumen und Gemüse), die zusammen mit den linearen Strukturen der Obstwiesen mit alten Apfelbäumen ein besonders ansprechendes Landschaftsbild ergeben. Alina berichtet dazu : „Während ich an dieser Schnittstelle zwischen Stadt und Land arbeitete, war ich immer wieder fasziniert von dieser räumlichen Konstellation. Oft fragte ich mich, was wohl die Leute in der U-Bahn dachten, wenn sie uns dort auf dem Feld sahen. Erkannten sie überhaupt, was wir ernteten? Konnten sie einen Bezug zwischen unserer Arbeit und ihrem Essen herstellen? Waren sie neidisch oder waren sie froh, dass sie diese Arbeit nicht tun mussten? Mir gefällt, dass Menschen dort vorbeigehen oder -fahren und diese „belebte“ Landwirtschaft, in der tatsächlich Menschen auf dem Acker arbeiten, zu sehen bekommen."

Abbildung 3. Rohrer Weg, die landschaftliche Vielfalt der Obst- und Gemüseparzellen des Reyerhofes in direkter Nachbarschaft zum Wohnviertel

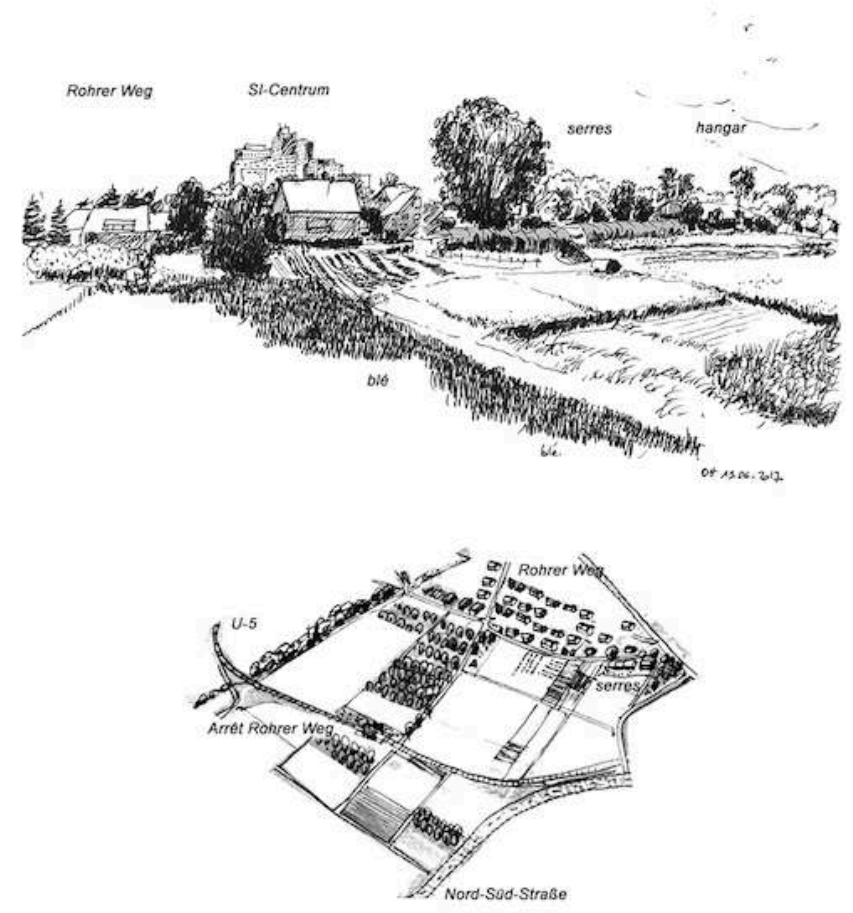

Dominique Henry, 2017 
Der Reyerhof bestimmt diese Mischkultur am Rande der Wohnviertel stark mit: der Betrieb baut neben $600 \mathrm{~m} 2$ Gewächshausfläche (unbeheizte Folientunnel) verschiedenes Feingemüse wie Salat, Tomaten, Zucchini etc. an. Dieses hat den höchsten Bedarf an täglicher Pflege (Bewässerung, häufige Ernten etc.). Diese Gemüsefelder stehen in engstem funktionalem Zusammenhang mit den Hofgebäuden : sie liegen dem Hof am nächsten und verfügen über eine Wasserentnahmestelle für die Bewässerung, außerdem befindet sich dort auch die Maschinenhalle.

\section{Vaihinger Straße}

Abbildung 4. Vaihinger Straße. Reizvolle Kontraste zwischen den weiten Kohl- und Getreidefeldern, die von Radwegen durchquert werden, und dem Stadtrand

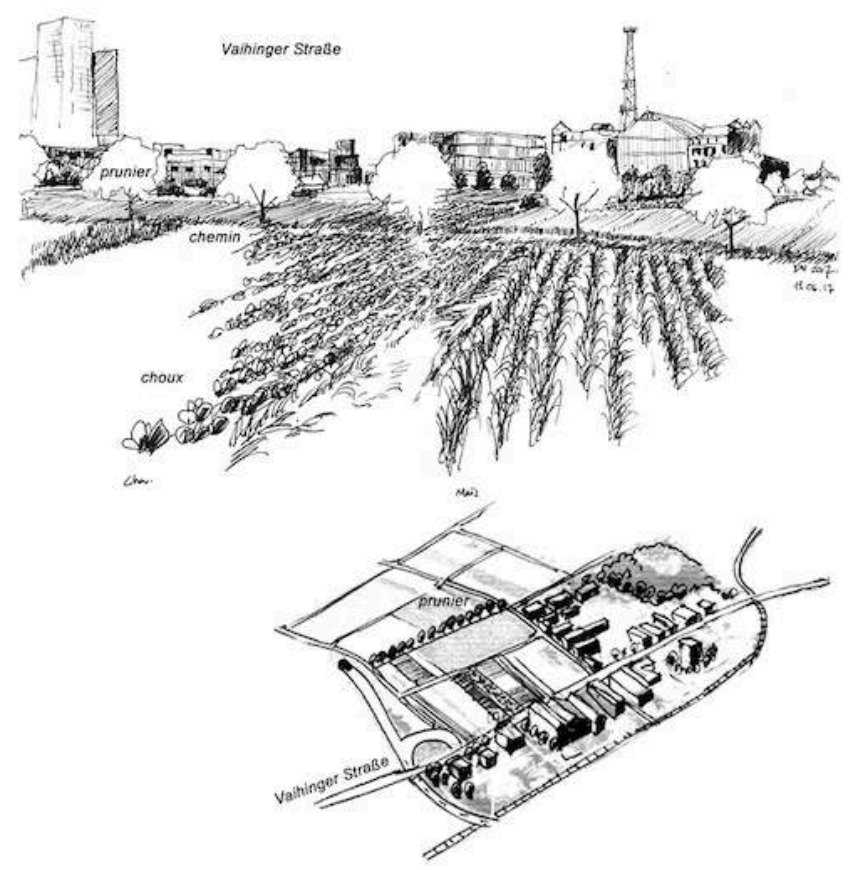

Dominique Henry, 2017.

Unweit davon bietet das Flurstück Vaihinger Straße ein ganz anderes Landschaftsbild. Dies liegt gleichermaßen an den hier auf größeren Parzellen angebauten Kulturen wie an der dahinter aufsteigenden Stadtkulisse. Im Süden, entlang der breiten Straße, befindet sich ein Komplex aus Dienstleistungsgebäuden mit 6 bis 10 Stockwerken, aus dem das Bauwerk von Gottfried Böhm ${ }^{8}$ mit seiner erstaunlichen Architektur heraussticht. Auf der Ostseite wird die Stadtkulisse dieses Flurstücks von kürzlich erbauten Wohnanlagen geprägt, deren kammförmiger Grundriss weite Ausblicke über die landwirtschaftlichen Flächen eröffnet. Nur ein asphaltierter Zugangsweg bildet den Übergang zwischen den Kulturen und den Gebäuden. Dieser Weg ist sehr verkehrsreich, denn hier sind viele landwirtschaftliche Fahrzeuge unterwegs, aber vor allem Fußgänger und Radfahrer, die zu bestimmten „Stoßzeiten“ ein beachtliches Verkehrsaufkommen verursachen. 
Zwischen der Reihe Pflaumenbäume und der Vaihinger Straße baut der Reyerhof hier je nach Fruchtfolge Kohl, Weizen, Möhren, Kartoffeln und Zwiebeln an. Befragt man die Landwirte dazu, was für sie eine solche Nähe zur Stadt bedeutet, so sehen sie sowohl positive Seiten wie auch Unannehmlichkeiten. So ist es nicht immer einfach, die viel befahrenen Straßen mit den Landmaschinen zu überqueren. Ebenso fällt es bisweilen schwer, die landwirtschaftlichen Erfordernisse und das Stadtleben unter einen Hut $\mathrm{zu}$ bringen, beispielsweise wenn bei trockenem Boden geerntet werden soll. Alina erinnert sich noch an die Lehmwolke, die im Herbst 2016 von der Kartoffelsortiermaschine aufgewirbelt und vom Wind dann zu den Fahrzeugen auf der Vaihinger Straße geweht wurde. Lukas berichtet auch davon, dass manchmal einige Reihen unerlaubt abgeerntet werden. Aber die Nähe zur Stadt kann auch positive Auswirkungen haben, insbesondere auf die Bevölkerung, die manchmal mit Staunen die Realität der landwirtschaftlichen Arbeit wahrnimmt, die hier für alle sichtbar - um nicht zu sagen im Rampenlicht - ausgeführt wird. Eine Angestellte erzählt von einem Einsatz zur Möhrenernte, der aufgrund von Regenvorhersagen an einem Feiertag durchgeführt wurde: „Viele Spaziergänger hielten inne, um die Arbeit des Möhrenvollernters zu beobachten, um den Traktor mit seinem Anhänger anzuschauen und die große Menschengruppe, die wir auf dem Feld bildeten : Angestellte, Praktikanten, Mitglieder der SoLaWi. Ein älteres Paar blieb stehen und wunderte sich über „all die jungen Leute", die bei der Ernte mitarbeiteten."

\section{Im Ried}

Abbildung 5. Im Ried bilden die vielfältigen vom Reyerhof angebauten Kulturen und die vielen kleinen Parzellen eine Landschaft im menschlichen Maßstab am Rand von Möhringen, einer Kleinstadt mit 31.000 Einwohnern

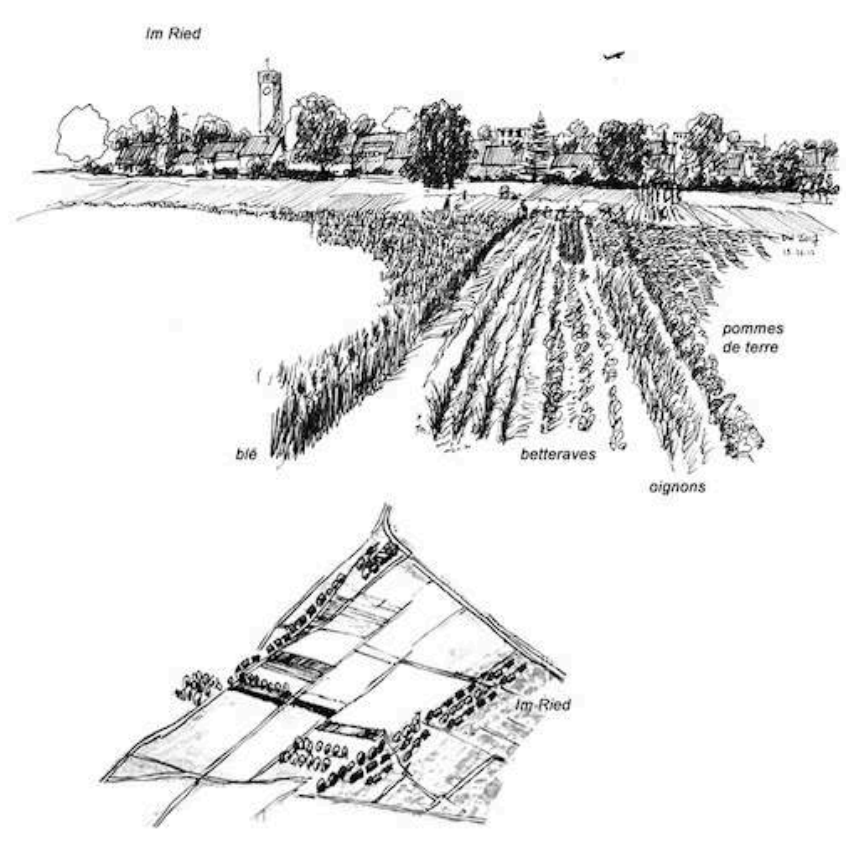


Die im Norden der Gemeinde bewirtschafteten Ackerflächen entsprechen am besten der Vorstellung einer landwirtschaftlich genutzten Ebene. Die Landschaft hat kein spürbares Relief. Sie ist im Norden wie im Süden von der dörflichen Silhouette der aus Einfamilienhäusern bestehenden Wohngebiete mit den zahlreichen Gärten und ihrer abwechslungsreichen Vegetation belebt. Letztere greift zum Teil die Baumschulen mit ihren Bäumen und Sträuchern auf, die über den Felder-Komplex verstreut liegen und von einigen verbliebenen Reihen Apfelbäume unterbrochen werden. Unter den Anbauflächen fallen die Parzellen des Hofes durch ihre Reihen mit Zwiebeln, Roter Bete, Möhren und Kartoffeln auf, die Rhythmus und Vielfalt in das Landschaftsbild bringen. Die Landwirte vom Reyerhof sind sich dessen bewusst, was die biologischdynamische Wirtschaftsweise, die auf Wechselbeziehungen und Artenvielfalt beruht, in Bezug auf die Landschaft bedeutet. „Gemäß der achtjährigen Fruchtfolge wird jedes Jahr eine andere Kultur auf dieser Parzelle angebaut. Dies sorgt nicht nur für gesunde Böden und weniger Schädlinge, sondern auch jährlich für ein neues Erscheinungsbild im landschaftlichen Gefüge“, betont Lukas. Alina schildert ihre Erfahrungen in Bezug auf Landschaften ebenso : „Mir wird vor allem auf Bahnreisen durch das Land bewusst, wie sehr Landwirte die Landschaft prägen. Die Agrarwüsten anderorts führen mir vor Augen, welche enorme Leistung der Reyerhof als vielfältiger Gemischtbetrieb auf den kleinparzellierten Flächen hier erbringt.“

\section{Fasanenhof}

Abbildung 6. Fasanenhof : Grünlandflächen zwischen Geschäftsviertel (links) und großem Wohnkomplex (rechts), die vom Hof gemäht werden

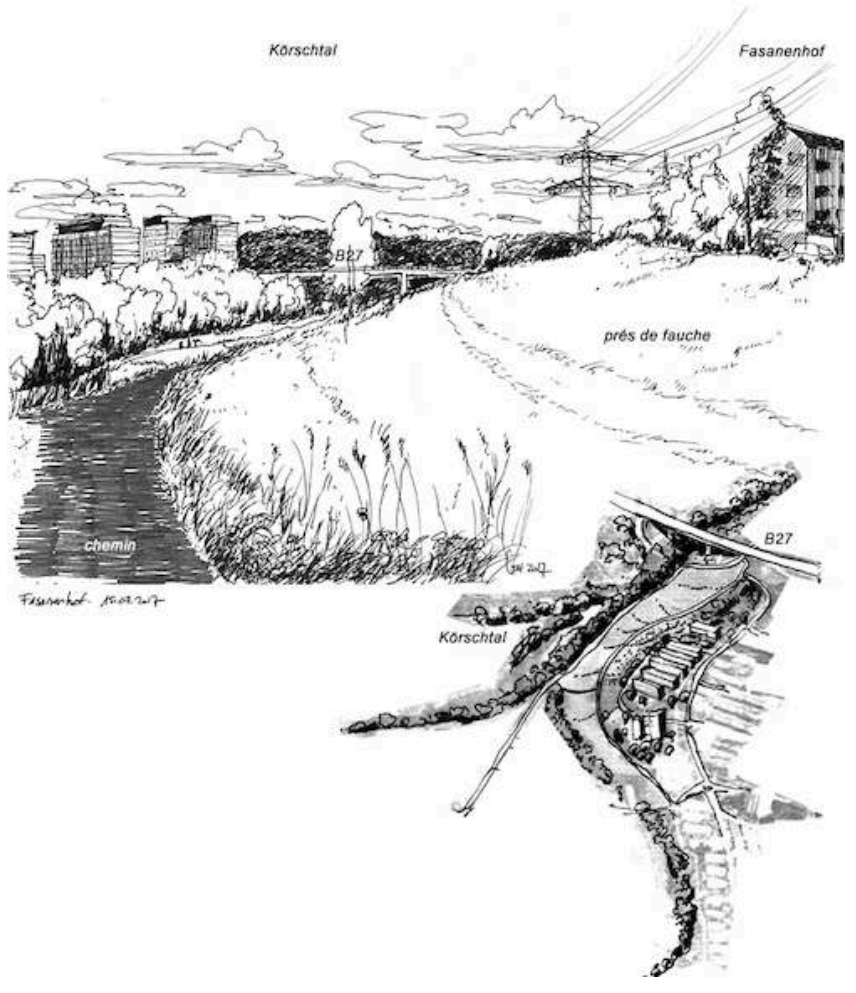

Dominique Henry, 2017. 
Im Süden bietet das Flurstück Fasanenhof ein ganz anderes Bild auf meinem Rundgang. Die vom hügeligeren Relief des Körschtals geprägte Landschaft wird von der Straßenbrücke der B27 (eine Zubringerstraße Richtung Stadtzentrum von Stuttgart) durchkreuzt und am Horizont von Hochhäusern überragt. Dabei handelt es sich auf der linken Seite des Bachs um Bürogebäude, während Fasanenhof auf der rechten Bachseite einer Großsiedlung aus Wohnblöcken und Hochhäusern entspricht, die nach den Grundsätzen des modernen Städtebaus errichtet wurden. Zu Fuße dieser Anlage, am Hang und im Talgrund, liegen die ausgedehnten Mähwiesen, die zum Teil vom Reyerhof genutzt werden. Das dort geerntete Heu ist Teil des Futtervorrats, der für die Winterfütterung der Milchkühe des Betriebs benötigt wird.

Hier ist bemerkenswert, wie stark die landwirtschaftlich genutzten Flächen in die Gesamtkomposition des Viertels integriert sind. Die Fassaden der Wohnblocks sind weithin zu dem kleinen Tal geöffnet. Starke Raum - und Verkehrskontinuen wurden entworfen und angelegt, sodass die Alleen und Rasenflächen am Fuße der Gebäude mit den Feldwegen und Wiesen des landwirtschaftlichen Raums zusammentreffen.

\section{Im Hof herrscht reges Treiben}

Abbildung 7. Im Herzen des alten Dorfkerns von Möhringen liegt der Reyerhof : unerwartet, außergewöhnlich und sehr belebt

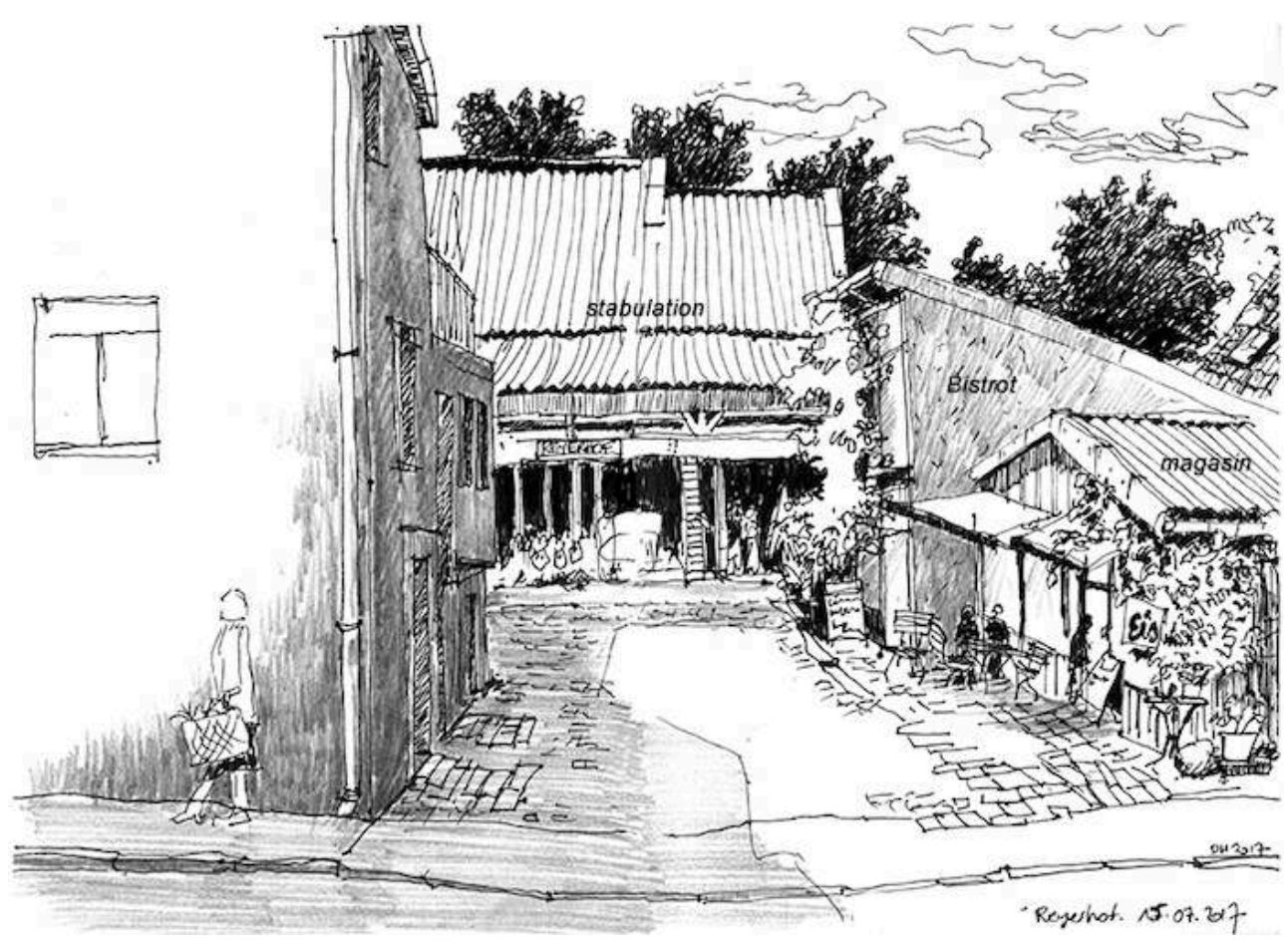

Dominique Henry, 2017.

Auf dieser Dorfstraße, wo der Kirchturm emporragt und sich niedrige Häuser mit kleinen, 2- bis 3-stöckigen Wohnbauten abwechseln, kündigt eigentlich nichts die Gebäude des Bauernhofes an, bevor sich die Freifläche des U-förmigen Hofes im rechten Winkel zur Straße hin öffnet. Und da: Überraschung! Hier herrscht geschäftiges Treiben wie auf einem kleinen Dorfplatz. Kunden betreten und verlassen den Hofladen, gehen wieder oder treffen sich mit anderen Personen an den Tischen des 
Bistros $^{9}$. In Hofladen und Bistro werden Produkte vom Hof verkauft, unter den neugierigen Blicken der zehn Kühe, die in ihrem Stall hinten im Hof gemächlich wiederkäuen. Je nach Tageszeit kommen dazu noch die Durchfahrten von Traktor und Lieferwagen sowie das Kommen und Gehen der Angestellten und Lehrlinge die, bevor sie wieder mit dem Fahrrad aufs Feld fahren, im linken Flügel dieses komplexen landwirtschaftlichen Gebäudeensembles ihre Mahlzeit einnehmen - und zum Teil auch dort wohnen. In den Gebäuden befinden sich zudem Büro, Küche, ein gemeinschaftliches Esszimmer ${ }^{10}$ und im Untergeschoss der Melkstand sowie die Räumlichkeiten für die Milchverarbeitung.

Es wird immer seltener, landwirtschaftliche Gebäude mit laufendem Betrieb inmitten eines alten Dorfkerns $\mathrm{zu}$ finden. Das bedeutet für diesen Bauernhof einen unbestreitbaren Geschäftsvorteil. Die Kühe sind eine von sämtlichen in der Umgebung lebenden Kindern (und nicht nur von diesen) geschätzte Attraktion. Der Besuch bei diesen Tieren bietet oftmals auch Gelegenheit, ein aus ihrer Milch hergestelltes Eis zu essen. Aber die Lage erfordert seitens des Landwirts auch in verschiedener Hinsicht eine Anpassung an die Nachbarschaft (Arbeitszeiten, Sauberkeit der Straße) und eine gewisse Geschicklichkeit, um Traktor und Anhänger durch eine enge Straße voller parkender Autos zu lenken.

\section{Vielfältigkeiten}

Am Ende meines Rundgangs ums Dorf bin ich überrascht von einer solchen Vielfalt. Da ist zum einen die Vielfalt der Agrar - und Stadtlandschaften, die je nach Flurstück sehr unterschiedlich sind. Und zum anderen die Vielfalt der Kulturen und der Vermarktungsweisen auf diesem Bauernhof, dem es scheinbar gelungen ist, einen Vorteil aus der urbanen Lage $\mathrm{zu}$ ziehen. Denn der Reyerhof versammelt Gemüseproduktion (4 ha), Getreideerzeugung und Ackerbau (15 ha) sowie Grünland (17 ha, davon 8 ha auf Streuobstwiesen) auf einer Gesamtfläche von 36 ha und hält außerdem 10 Milchkühe, was die Arbeitskraft von 7 Personen erfordert. Die Produktion wird vollständig direkt vermarktet (nachdem ein Teil der Milch zu Frischkäse, Joghurt und Eis verarbeitet wurde), und zwar im Hofladen und im Bistro, wo 10 Angestellte arbeiten (dies entspricht 5 Vollzeitkräften), und über die wöchentliche Lieferung von 280 Ernteanteilen ${ }^{11}$ für die SoLaWi Stuttgart. Die Vermarktung der Erzeugnisse setzt somit auf die große Zahl an potenziellen Verbrauchern in der näheren Umgebung ${ }^{12}$ sowie auf ein Qualitätskonzept, das auf vielerlei Ebenen zu finden ist: Qualität der Lebensmittelerzeugung gemäß den Richtlinien der biologisch-dynamischen Wirtschaftsweise (Demeter) seit $1957^{13}$, Qualität und Pflege der landwirtschaftlichen Architektur und ihres Umfelds, und schließlich Qualität und Vielfalt der Landschaften in unmittelbarer Nachbarschaft zu Geschäfts - und Wohnvierteln. Das System ist vielseitig und komplex. Es handelt sich um einen Landwirtschaftsbetrieb, der immer städtischer geworden ist durch seine Interaktion mit den Stadträumen und mit den Bewohnern der Stadt, insbesondere den Mitgliedern der SoLaWi.

Eine Weiterführung der Forschung wäre natürlich wünschenswert, um Antworten auf die Fragen $\mathrm{zu}$ erhalten, die der vorliegende Bericht aufwirft. Wie beeinflusst (oder nicht) beispielsweise dieser enge Kontakt zwischen Landwirtschaft und Stadt die Methoden der Landwirte auf dem Reyerhof und die Anbauweisen? Welche Auswirkungen hat dies auf die Organisation der landwirtschaftlichen Arbeit? 
Beeinflusst diese Sichtbarkeit der Ackerflächen im Landschaftsbild, vergleichbar mit einem öffentlichen Raum innerhalb des Stadtgefüges, den Landwirt bei der Auswahl der Techniken und in seinem Bewusstsein für die Landschaft? Und im Gegenzug, sind sich die SoLaWi-Mitglieder dieser erstaunlichen Verflechtung des Reyerhofes mit SuttgartMöhringen bewusst? Aus welchen Beweggründen unterstützen sie diese Landwirtschaft und diesen Bauernhof?

Dabei ginge es außerdem darum, die Überlegungen auf eine breitere Grundlage zu stellen und Vergleiche mit anderen Landwirtschaftsbetrieben und anderen Großstadtlagen anzuregen.

\section{Großstadt sucht Annäherung an die Landwirtschaft - und umgekehrt}

Am Ende dieser Erkundungen der Landwirtschaft auf stadtnahen Flächen scheint es mir notwendig, einige Überlegungen als vorläufige Schlussfolgerung darzulegen.

\section{Wenn die Stadt näher heranrückt... aber von welcher urbanen Dynamik ist die Rede?}

Hier muss vielleicht noch einmal auf den Wandel des Bauernhofes und seiner Produktionsfläche eingegangen werden : von einem familiengeführten Hof mit Flächen rund um das Dorf Möhringen, hin zu einem Landwirtschaftsbetrieb inmitten des Stadtgebiets von Stuttgart-Möhringen, geführt von einem landwirtschaftlichen Kleinunternehmen, das Lebensmittel erzeugt, verarbeitet sowie im Direktverkauf und zum Teil auf solidarischer Basis vertreibt. Diese Landwirtschaft ist somit zu einer städtischen geworden, weil die Stadt näher gerückt ist. Aber dieses Näherrücken kann man unterschiedlich sehen. Zum einen die räumliche Annäherung, die erduldet ist, wenn die Verstädterung vordringt und Flächen entzieht; und zum anderen die soziale Annäherung, die ihrerseits neue Perspektiven für Zusammenarbeit und Austausch eröffnet.

Die erstgenannte urbane Dynamik ist wohlbekannt: sie schluckt die Flächen oder verfolgt andere Projekte, manchmal auch umweltbezogene, ohne dass jedoch der Landwirtschaft besonderes Augenmerk zukommt. Das Geschehen rund um die Flächen am Rohrer Weg ist dafür ein aufschlussreiches Beispiel. Die Geschichte beginnt im März 2002, als die Stadträtinnen und Stadträte (CDU, FDP und Freie Wähler) von Möhringen beim Gemeinderat eine Änderung des Flächennutzungsplans beantragen, um den Anteil an Bauland am Rohrer Weg zu erhöhen. Am 26. Juni genehmigt der Gemeinderat das Bauvorhaben gegen die Stimmen von Grünen und SPD. Daraufhin wird am Folgetag, dem 27.Juni, sofort der Verein „Rettet die Felder und Streuobstwiesen am Rohrer Weg ${ }^{{ }_{14}}$ gegründet. Der Verein initiiert eine Unterschriftenaktion, eine Bürgerinitiative sowie die Besichtigung der Streuobstwiesen durch einige bedeutende Politiker. Nach diversen politischen Wechselfällen wird die gesamte Fläche den städtebaulichen Fragen entzogen, indem sie im August 2005 unter Landschaftsschutz gestellt wird. Das Hauptargument dabei lautet... der Vogel - und Klimaschutz ${ }^{15}$. Die Wahrung des Produktionspotenzials der Felder und Streuobstwiesen scheint nicht die entscheidende Motivation gewesen zu sein, selbst wenn in gewisser 
Weise die Proteste und die ökologischen Bestimmungen das Fortbestehen dieses landwirtschaftlichen Raumes ermöglicht haben.

Die zweite Dynamik ist diejenige, die eine Gruppe von Stadtbewohnern dazu bewogen hat, sich dem Bauernhof anzunähern. Und nicht umgekehrt. In der Tat haben sich zuerst einige engagierte Verbraucher mit einem ethischen Bewusstsein für ihre Ernährung auf die Suche nach einem Landwirtschaftsbetrieb „in der Stadt“ begeben, der bereit wäre, mit ihnen ein solidarisches Konzept aufzubauen. So betont Alina, die von Beginn an bei diesem Abenteuer mitgewirkt hat: „Die Produktion sollte für uns einsehbar sein und lange Transportwege aus ökologischer Überzeugung vermieden werden. Darüber hinaus bestand der starke Wunsch, einen persönlichen Kontakt zu dem Hof zu pflegen und dort auch mitzuarbeiten. Ein kontinuierlicher Austausch zwischen Erzeuger und Verbraucher sollte die gemeinsame Entwicklung des Projekts ermöglichen. Ziel war es also, einen stadtnahen, ökologisch wirtschaftenden Betrieb zu finden.“ Und sie führt weiter aus, was die Verbraucher zum Reyerhof geführt hat : „Am Ende gab es drei potenzielle Höfe, von denen einer tatsächlich aufgrund der Entfernung (ca. $30 \mathrm{~km}$ ) ausschied. Die anderen beiden waren beide ähnlich stadtnah und der Reyerhof wagte schließlich als Erster das Experiment mit uns. Die Stadtlage und Erreichbarkeit mit den öffentlichen Verkehrsmitteln haben das gegenseitige Kennenlernen und die gemeinsame Planung sehr erleichtert.“

Abbildung 8. Landschaft, die stadtnah Nahrungsmittel produziert ; Stuttgart-Möhringen

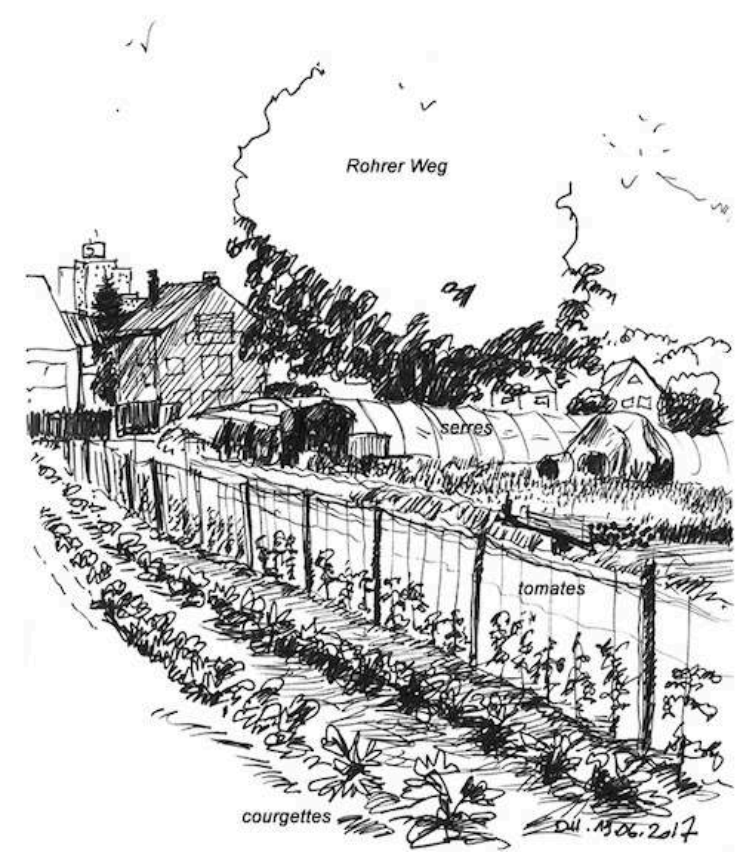

Dominique Henry, 2017.

Die anfängliche Annäherung der Stadtbewohner an den Reyerhof hat sich dann zu einer gegenseitigen Bindung entwickelt. Diese hatte vonseiten des Bauernhofes beispielsweise eine Anpassung der Gemüseproduktion zur Folge. Denn für die Zusammenstellung der wöchentlichen anteilsmäßigen Lieferung hat der Bauernhof die 
von den Verbrauchern geäußerten Wünsche berücksichtigt, indem er zum Beispiel das Gemüsesortiment erweitert hat und indem die Produktherkunft noch stärker garantiert wird : das ist beispielsweise der Fall bei dem Mehl, für das ausschließlich auf dem Reyerhof angebauter Weizen verwendet wird und mit dem ein benachbarter Bäcker spezielle Hofbrote bäckt, die immer donnerstags im Laden und in den SoLaWiSAnteilen erhältlich sind. Lukas prüft außerdem die Möglichkeit, Roggen anzubauen, um das Brotangebot zu ergänzen.

Die Solidarwirtschaft, auf deren Basis die SoLaWi funktioniert, gibt dem Landwirt die Garantie, seine Produktion - mehr oder weniger ertragreich - zu einem fairen Preis zu verkaufen, der im Voraus bei einer Jahresversammlung der Mitglieder festgelegt wurde, bei der auch das Budget verabschiedet wird. Zweimal haben die Mitglieder der SoLaWi, die bei dieser Gelegenheit Einsicht in die Bücher haben, den Wunsch geäußert, dass der Stundenlohn der Hofangestellten erhöht werde. Durch Abstimmung hat die Gruppe beschlossen, die Kosten dafür durch eine Erhöhung ihrer Anteile zu tragen.

Im Endeffekt kann die geografische Nähe zur Stadt, die in mancher Hinsicht Einschränkungen mit sich bringt, auch ein Vorteil sein. Allerdings nur dann, wenn man es versteht, daraus Nutzen zu ziehen.

\section{Ein biologisch-dynamisches Landwirtschaftsprojekt}

Nun noch zu einem letzten Punkt, der für die Landwirtschaft von zentraler Bedeutung ist, und noch mehr für Landwirtschaft in der Stadt : die Frage nach Grund und Boden. „Raum ist ein knappes Gut, und zahlreiche Akteure sind an den Flächen interessiert, seien sie bebaut oder unbebaut, und folglich sind die Preise für Boden hoch", wie Alina betont.

Die Reaktion vonseiten des Bauernhofes in Bezug auf Boden ist diesbezüglich sehr interessant. Seine landwirtschaftliche Nutzfläche beträgt 36 ha, von denen außer einem einzigen Hektar alles Pachtgrundstücke sind. 1969 lag sie bei 20 ha und 1998 bei 29 ha. Die Zunahme ist kontinuierlich, aber gemäßigt. Sie bleibt unter dem Durchschnitt (alle Betriebe zusammengenommen), der in Deutschland wie in Frankreich bei ca. 61 ha liegt ${ }^{16}$. Sie ist auch weit entfernt von der exzessiven Vergrößerung, wie sie von einigen landwirtschaftlichen Betrieben beworben wird, die damit nach wirtschaftlicher Rentabilität streben. Das hier angewandte Konzept besteht in der Optimierung der verfügbaren Flächen : Der geringe Zuwachs an Anbauflächen wird durch einen starken Anstieg der Wertschöpfung bei den landwirtschaftlichen Produkten korrigiert. Aber dieses Konzept betrifft nicht nur Grund und Boden. Es erfordert letzten Endes die Fähigkeit des Landwirtschaftsprojekts, lebendig und dynamisch zu sein - und zu bleiben - vielleicht eine andere Definition der biologisch-dynamischen Landwirtschaft. D.h. in der Lage zu sein, sich anzupassen und zwischen Einschränkungen einerseits und Vorteilen andererseits auf allen Ebenen Nutzen zu ziehen aus seiner städtischen Einbindung.

Das biologisch-dynamische Landwirtschaftsprojekt des Reyerhofes, wie es in den Agrarund Stadtlandschaften von Möhringen sichtbar ist, bietet sicher einigen fruchtbaren Boden.

Ich möchte mich an dieser Stelle ganz herzlich bei Alina bedanken, deren schriftlicher Text für mich von großem Nutzen war, bei Lukas für seine konkreten und praktischen Antworten, bei 
Katrin für ihre wertvolle Hilfe bei der Übersetzung, sowie bei allen Lebewesen des Reyerhofes, die mich regelmäßig mit köstlichen Produkten versorgen.

\section{BIBLIOGRAPHIE}

Ambroise, R., Bonneaud, F., Brunet-Vinck, V., Agriculteurs et Paysages. Dix exemples de projets de paysage en agriculture, Dijon, Educagri, 2000, $207 \mathrm{~S}$.

Augé, M, La Vie en double, Paris, Payot et Rivages, 2011, 268 S.

Donadieu, P., Campagnes urbaines, Arles, Actes Sud, 1998, 224 S.

Bernetti, I., Fanfani, D., Monacci, F., Poli, D., Rubino, A., „Le parc agricole comme instrument de l'aménagement stratégique multifonctionnel des espaces périurbains de la Toscane centrale“, Internationales Kolloquium „Les agricultures périurbaines, un enjeu pour la ville. Vers des projets de territoire“, Nanterre (Frankreich), 10.-12. Oktober 2007.

Birre, A, „Chez M. Karl Reyer. Petite ferme allemande de Möhringen“, in Une politique de la terre, Marcq-Lille, Éditions Vie et Action, 1969, S. 207-209.

Boutleux, D., „Les champs urbains. Réinventer les mythologies urbaines“, Openfield, $\mathrm{n}^{\circ} 2$, Juni 2013, URL : https://www.revue-openfield.net/2013/06/23/les-champs-urbains-reinventerles-mythologies-urbaines/.

Deffontaines, J.-P., Les Sentiers d'un géoagronome, Paris, Arguments, 1998, 360 S.

Étienne, M, „Voyage à Kraftwerk“, Criticat, nº 11, 2013, S. 13-23

Henry, D., „Un voyage paysagiste en agriculture“, Revue d'Auvergne, vol. „Des paysages pour le développement local. Expériences et recherches innovantes dans le Massif central“", $n^{\circ}$ 571, 2004, S. 115-131.

Janin, R., La Ville agricole, Pantin, Éditions Openfield, 2017, 70 S.

Janin, R., „Trois enclaves agricoles dans la périphérie de Lyon, vers des agricultures urbaines ?“, Projets de paysage, $\mathrm{n}^{\circ}$ 2, Juni 2009, online : http://www.projetsdepaysage.fr/ trois_enclaves_agricoles_dans_la_p_riph_rie_de_lyon_vers_des_agricultures_urbaines_.

Laverne, T., „L'expérience du Triangle vert, projet de territoire agri-urbain durable“, Cahier thématique. Agriculture métropolitaine/Métropole agricole, 2011, n 11, ENSAP de Lille, S. 257-271.

Masboungi, A. (dir.), Extension du domaine de l'urbanisme. Frédéric Bonnet, Grand Prix de l'urbanisme 2014, Paris, Parenthèses, 2014, 144 S.

\section{NOTES}

1. http://www.trianglevert.org/

2. Vgl. dazu auch den Artikel im Themendossier dieser Ausgabe Nr. 17 von Projets de paysage, hier klicken : https://journals.openedition.org/paysage/4782

3. https://www.reyerhof.de/ 
4. Ich möchte an dieser Stelle darauf hinweisen, wer mich zu meiner Erkundung rund um den Reyerhof inspiriert hat: es ist der Architekt und Illustrator Martin Étienne mit seinem Werk „Voyage à Kraftwerk“ („Reise nach Kraftwerk“), einem gezeichneten Besuch dieser Wohngenossenschaft, erschienen 2013 in der Zeitschrift Criticat, - allerdings während der schönen Jahreszeit! : http://jhapa.eu/wp-content/uploads/2016/09/criticat11_MartinEtienne.pdf

5. In dem Sinne, wie Marc Augé (2011) beschreibt, auf Deutsch etwa: „Die Begegnung suchen, das könnte im Gegenteil bedeuten, sie zu erschaffen, sie zu gestalten, etwas aus dem zu machen, das nichts als bloßer Zufall war. Jeden Tag laufe ich tausend Menschen über den Weg, aber ich begegne ihnen nicht. Wenn ich ihnen begegne, dann deshalb, weil etwas in ihnen meine Aufmerksamkeit erregt hat, weil sie mir „etwas sagten“, und weil letztendlich ich aus nichts etwas machen wollte, aus dem Zufall ein Ereignis machen und eine unbekannte Person erkennen wollte."

6. Für „Solidarische Landwirtschaft“. Dies entspricht in Frankreich den AMAP („Association pour le maintien d'une agriculture paysanne“, auf Deutsch etwa „Verbrauchervereinigungen für die Erhaltung bäuerlicher Landwirtschaft"). Weitere Informationen: https://www.solidarischelandwirtschaft.org/de/startseite/

7. Ober - und unterirdische Stadtbahn mit den charakteristischen gelben Zügen, deren Netz den gesamten Ballungsraum umfasst.

8. Zeitgenössischer deutscher Architekt, geboren 1920, der mit zahlreichen Preisen ausgezeichnet wurde, darunter 1986 mit dem angesehenen Pritzker-Preis.

9. So nennt sich das Restaurant, in dem ein Teil der Hoferzeugnisse verarbeitet wird.

10. Die Mahlzeiten (Frühstück und Mittagessen) werden dort gemeinsam eingenommen.

11. Stand 2018: 360 Anteile.

12. Hier sei darauf hingewiesen, dass die Gemeinde Möhringen, einer der 23 Stadtbezirke von Stuttgart, 31.000 Einwohner zählt (von 610.000 Einwohnern des Ballungsraums).

13. Diesbezüglich kann man den interessanten Erlebnisbericht von André Birre lesen, der $1969 \mathrm{zu}$ Karl Reyer kam, um einen „kleinen deutschen Bauernhof in Möhringen“ zu besichtigen: „Herr Reyer hat vor 12 Jahren mit der biologisch-dynamischen Wirtschaftsweise begonnen, nachdem er bei einem Vortrag gewesen war und weil er auf seinem Hof die Nachteile der chemischen Düngung gespürt hatte.“

14. Für weitere Informationen siehe die vollständige Chronologie, wie sie vom Verein auf seiner Website dargestellt wird: http://rohrer-weg.de/Chronologie/chronologie.html

15. Denn das Gebiet gilt als „Kaltluftschneise“ und leistet somit einen Beitrag zur Regulierung des Stadtklimas in Bezug auf den Kessel, in dem das Stadtzentrum Stuttgarts liegt.

16. http://ec.europa.eu/eurostat/statistics-explained/index.php/Farm_structure_statistics/fr

\section{RÉSUMÉS}

„Landwirtschaft in der Stadt für die Stadt" : so lautet der Werbespruch, den der Reyerhof, ein biologisch-dynamischer Gemischtbetrieb, gewählt hat. Dieser Spruch dient der Werbung und drückt zugleich einen tatsächlichen Sachverhalt aus. Denn die vom Reyerhof bewirtschafteten Parzellen liegen inmitten des Stuttgarter Stadtgebiets. Sie grenzen direkt an die bebaute Fläche an oder fügen sich dazwischen ein. Der Autor - Landschaftsarchitekt und Zeichner - lädt zu einer 
Erkundung der landwirtschaftlich genutzten Flächen im Stadtgebiet ein, um die Agrarlandschaften und diejenigen, die diese Flächen bewirtschaften, kennenzulernen. In diesem Beitrag soll es darum gehen, einige landschaftliche Gegebenheiten dieses Landwirtschaftsbetriebs in der Großstadt zu erfassen, zu beschreiben und darzustellen. Das hier zusammengetragene Material könnte Ausgangspunkt für eine breiter angelegte Untersuchung sein.

«L'agriculture dans la ville, pour la ville » est le slogan adopté par le Reyerhof, une ferme en polyculture-élevage biodynamique. Ce slogan traduit certes un argument commercial mais aussi un état de fait. Les parcelles que celle-ci travaille s'inscrivent en effet dans le territoire urbanisé de Stuttgart. Elles occupent la lisière des quartiers ou alors s'intercalent entre eux. L'auteur, qui est paysagiste et dessinateur, convie à un voyage en terres urbaines cultivées à la rencontre des paysages agricoles et de ceux qui les cultivent. Saisir, décrire et représenter les paysages de cette exploitation agricole métropolitaine est le sens donné à cette contribution en rassemblant un peu de matières premières à ce qui pourrait potentiellement devenir un travail plus large de recherche et de projet.

\section{INDEX}

Schlüsselwörter : ländlicher Raum, städtischer Raum, Landwirtschaft, Großstadt, Landwirt, Verbraucher, Direktvermarktung, Landschaftsarchitekt, Stuttgart

Mots-clés : paysage agricole et urbain, agriculture, métropole, agriculteur, consommateur, vente directe, paysagiste, Stuttgart

\section{AUTEURS}

\section{DOMINIQUE HENRY}

Staatlich geprüfter Landschaftsarchitekt (DPLG, Frankreich) und promovierter Geograf, Dozent an der französischen Hochschule für Architektur und Landschaft (École nationale supérieure d'architecture et de paysage) Lille sowie Forscher an der staatlichen Forschungseinrichtung CNRS (Centre National de la Recherche Scientifique) in der gemischten Forschungseinheit

„UMR 5319 Passages“. Er arbeitet außerdem freiberuflich in seinem Landschaftsplanungsbüro „Le champ d'à côté“. In seinen Arbeiten und Forschungsprojekten interessiert sich Dominique Henry für Landschaftsentwicklung in landwirtschaftlich geprägten Gebieten. Sein Tätigkeitsfeld umfasst sowohl die Entwicklung ländlicher Räume im Großen als auch die Gartengestaltung im Kleinen.

lechampdacote[at]gmail[dot]com 